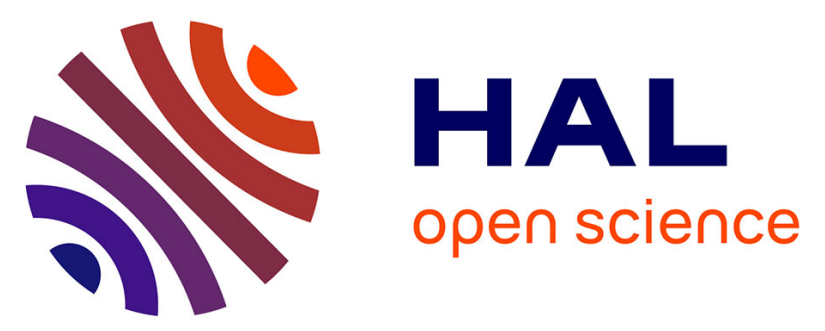

\title{
NON PARAMETRIC CELL NUCLEI SEGMENTATION BASED ON A TRACKING OVER DEPTH FROM 3D FLUORESCENCE CONFOCAL IMAGES
}

\author{
Thierry Pécot, Shantanu Singh, Enrico Caserta, Kun Huang, Raghu \\ Machiraju, Gustavo Leone
}

\section{To cite this version:}

Thierry Pécot, Shantanu Singh, Enrico Caserta, Kun Huang, Raghu Machiraju, et al.. NON PARAMETRIC CELL NUCLEI SEGMENTATION BASED ON A TRACKING OVER DEPTH FROM 3D FLUORESCENCE CONFOCAL IMAGES. ISBI - 9th IEEE International Symposium On Biomedical Imaging : from nano to macro - 2012, May 2012, Barcelona, Spain. hal-00921540

\author{
HAL Id: hal-00921540 \\ https://hal.inria.fr/hal-00921540
}

Submitted on 20 Dec 2013

HAL is a multi-disciplinary open access archive for the deposit and dissemination of scientific research documents, whether they are published or not. The documents may come from teaching and research institutions in France or abroad, or from public or private research centers.
L'archive ouverte pluridisciplinaire HAL, est destinée au dépôt et à la diffusion de documents scientifiques de niveau recherche, publiés ou non, émanant des établissements d'enseignement et de recherche français ou étrangers, des laboratoires publics ou privés. 


\title{
NON PARAMETRIC CELL NUCLEI SEGMENTATION BASED ON A TRACKING OVER DEPTH FROM 3D FLUORESCENCE CONFOCAL IMAGES
}

\author{
Thierry Pécot ${ }^{1,3}$, Shantanu Singh ${ }^{1,3}$,Enrico Caserta ${ }^{1}$, Kun Huang $^{4}$, Raghu Machiraju $^{3}$, Gustavo Leone Len $^{1,2}$ \\ ${ }^{1}$ Human Cancer Genetics Program, ${ }^{2}$ Department of Molecular Genetics, \\ ${ }^{3}$ Computer Science And Engineering, ${ }^{4}$ Biomedical Informatics, The Ohio State University, Columbus, Ohio
}

\begin{abstract}
3D cell nuclei segmentation from fluorescence microscopy images is a key application in many biological studies. We propose a new, fully automated and non parametric method that takes advantage of the resolution anisotropy in fluorescence microscopy. The cell nuclei are first detected in $2 \mathrm{D}$ at each image plane and then tracked over depth through a graph based decision to recover their 3D profiles. As the tracking fails to separate very close cell nuclei along depth, we also propose a corrective step based on an intensity projection criterion. Experimental results on real data demonstrate the efficacy of the proposed method.
\end{abstract}

Index Terms - Detection, tracking over depth, fluorescence confocal microscopy

\section{INTRODUCTION}

In cancer biology, the comprehensive study of cellular phenotypes provides information about the structure and function of different cell compartments (fibroblasts, macrophages, endothelial and epithelial cells) in the microenvironment. A detailed study of nuclear morphology will allow for the association of the phenotypes to specific genotypes (wild-type, mutants). This analysis needs to first segment the cell nuclei.

Many segmentation methods have been deployed on fluorescent microscopy images. Geometric active contours methods take advantage of the level sets framework to extract the cell nuclei boundaries by using an energy term defined from intensity variations. These methods have been successfully applied to 3D microscopy image stacks [1,2]. However, the success of these methods is very dependent on the initial selection of the contours. Watershed based methods [3, 4] consider images as topographic reliefs and segment objects of interest by releasing water from seed points. Unfortunately, these methods tend to over-segment cluttered cell nuclei. In [5], the authors propose a gradient diffusion procedure followed by a gradient flow tracking to localize cell nuclei centers. A local adaptive thresholding is then applied to achieve a segmentation of the cell nuclei. This method is particularly efficient for high quality and high resolution images.
In 3D fluorescent confocal images, the resolution along the depth is poorer than in the two other directions. This anisotropy prevents the robust capture of cell nuclei. To accommodate this disparity in the resolutions, we propose a new approach based on cell tracking and a graph based decision process. First, we detect cell nucleus components at each plane of the image volume. Then, we present a graph based decision process to track the cell nucleus components over depth. As the tracking over depth is not always able to separate cluttered cell nuclei, we also introduce a correction step that exploits an intensity projection criterion. We demonstrate the effectiveness of our approach by comparing its performances to competitive cell nuclei segmentation methods using real 3D fluorescence confocal images with both isolated and cluttered cell nuclei.

\section{CELL NUCLEI DETECTION}

Methods based on intensity local curvature [6], Laplacian of Gaussian [7], conditional random fields [8], mathematical morphology $[9,10]$ or image decomposition [11] for cell nuclei detection were reported earlier in the literature. In this study, we decide to divide this process into three steps: i) compute a detection measure; ii) threshold the detection measure; iii) identify each cell nucleus component.

For computing a detection measure, we choose the multiscale approach of [12] since it is robust to noise in fluorescence images and it enables to consider different image resolutions. This approach divides an input image into several wavelet planes at different scales. In practice, $K$ resolutions $\left\{\mathbf{I}_{z}^{(1)}, \ldots, \mathbf{I}_{z}^{(K)}\right\}$ of the original image intensity $\mathbf{I}_{z}^{(0)}$ acquired at depth $z$ are obtained through the repeated convolutions with a Gaussian separable kernel along the two canonical directions:

$$
\mathbf{I}_{z}^{(i)}=\mathbf{I}_{z}^{(i-1)} * G_{B 3}, \quad 1 \leq i \leq K,
$$

where $G_{B 3}=\left[\frac{1}{16}, \frac{1}{4}, \frac{3}{8}, \frac{1}{4}, \frac{1}{16}\right]$ is a B3-spline version of the isotropic wavelet transform [13]. The wavelet planes are then defined as the difference between two consecutive resolutions of the original image:

$$
\mathbf{W}_{z}^{(i)}=\mathbf{I}_{z}^{(i-1)}-\mathbf{I}_{z}^{(i)}, \quad 1 \leq i \leq K .
$$




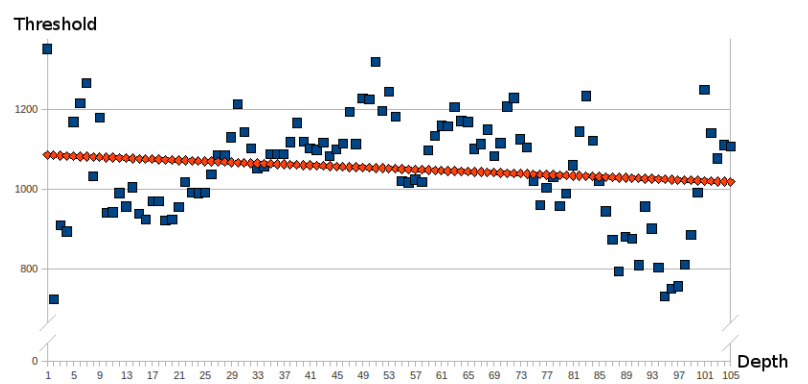

Fig. 1. Thresholds estimated with the Maximum Entropy Thresholding method on a stack of 105 images followed by a linear least squares estimation.

The lower the wavelet plane resolution is, the higher the image scale is.

To locate the cell nucleus components, we propose to use the Maximum Entropy Thresholding (MET) method [14] to threshold each wavelet plane. The MET method provides the threshold that maximizes the sum of the entropies measured in the object and background components. The thresholds estimated at each depth vary according to the intensity distribution in each image (see Fig.1). This distribution depends not only on the number of cell nucleus components in each image, but also on the attenuation arising from photobleaching. The thresholds have to be similar across the image planes to prevent from detection jumps: a part of a nucleus is detected at depth $z$, not detected at depth $z+1$ and detected again at depth $z+2$. In order to avoid this phenomenon and to mitigate the photobleaching effects, we propose to regularize the thresholds over the image stack by using a linear least squares fitting procedure (see Fig.1).

The final step for detection is the identification of each cell nucleus component at each depth. For this purpose, the connex components in the thresholded wavelet planes are associated to cell nucleus components. But, as two spatially close cells will only be associated to one connex component, we prefer to consider each local maximum in the wavelet planes located in these areas. Let $\mathbf{x}_{z}=\left\{x_{z}^{1}, \ldots, x_{z}^{r}\right\}$ be the set of $r$ local maxima at depth $z$ located in the thresholded wavelet plane regions. When two local maxima belong to the same component, they have to be separated. We propose to take advantage of the minimal path framework $[15,16]$ given its computational efficiency. It has to be noted that watershed methods lead to the same results. When $l$ local maxima $x_{z}^{j}, j=1, \ldots, l$ belong to the same cell nucleus component, we compute the minimal action maps associated to each local maximum:

$\forall x \in \mathcal{S}, \mathcal{U}_{x_{z}^{j}}(x)=\min _{\mathcal{A}\left(x_{z}^{j}, x\right)}\left\{\int_{\gamma\left(x_{z}^{j}, x\right)}\left(\mathcal{P}\left(\gamma\left(x_{z}^{j}, x\right)\right)(\ell)\right] d \ell\right\}$,

where $\mathcal{S}$ is the grid of pixels, $\mathcal{A}\left(x_{z}^{j}, x\right) \in \mathcal{S} \times \mathcal{S}$ is the set of planar curves that link $x_{z}^{j} \in \mathcal{S}, x \in \mathcal{S}$ are the points in the considered connex component, $\gamma\left(x_{z}^{j}, x\right) \in \mathcal{A}\left(x_{z}^{j}, x\right), \mathcal{P}$ : $\mathcal{S} \rightarrow \mathbb{R}$ is a potential that takes low values when $\mathbf{W}_{z}^{(i)}$ is

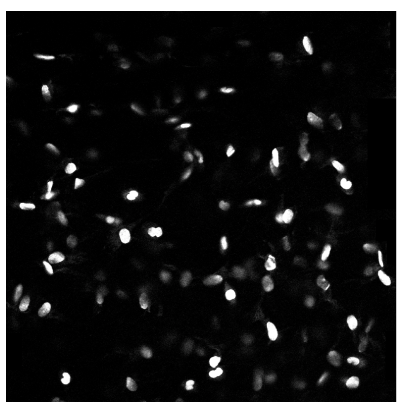

(a)

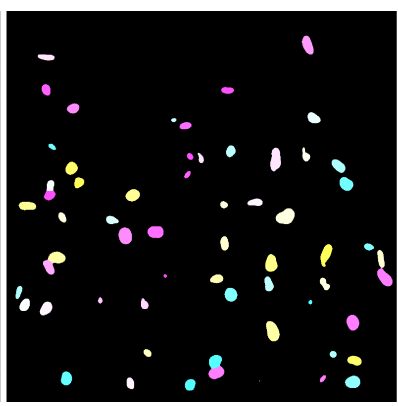

(b)
Fig. 2. (a) Confocal fluorescence image showing cell nuclei tagged with DAPI; (b) detected cell nucleus components where each color corresponds to one cell nucleus component.

high and $\ell$ is the curvilinear abscissa. The minimal action maps associated to each local maximum are computed with the fast marching algorithm [17]. The detection of individual cell components obtained on a real image image is illustrated in Fig.2.

\section{GRAPH BASED DECISION}

We propose to apply a tracking algorithm across image planes. By establishing a correspondence between the cell nucleus components along depth, the complete nuclei can be reconstructed.

All $r$ local maxima identified during the detection $\left\{x_{z}^{1}, \ldots, x_{z}^{r}\right\}$ at depth $z$ are associated to $r$ nodes $\left\{n_{z}^{1}, \ldots, n_{z}^{r}\right\}$. Each node $n_{z}^{u}$ at depth $z$ is linked to the nearest node $n_{z+1}^{v}$ at depth $z+1$ by an edge $e_{z+1}^{u v}$ (see fig.3 (b)). A cost $c_{z+1}^{u v}$ is attributed to the edge $e_{z+1}^{u v}$ and is equal to the distance between $x_{z}^{u}$ and $x_{z+1}^{v}$. Eventually, $T$ graphs $\mathcal{G}_{t}, t=\{1, \ldots, T\}$ corresponding to one or several cell nuclei are built. Let $o_{z}^{\mathcal{G}_{t}}$ be the number of nodes in $\mathcal{G}_{t}$ at depth $z$ and $o_{\max }^{\mathcal{G}_{t}}$ be the maximum number of nodes in $\mathcal{G}_{t}$ for a given depth. Let $\mathbf{1}(\cdot)$ be the indicator function such that:

$\mathbf{1}\left(o_{z}^{\mathcal{G}_{t}}=m\right)= \begin{cases}1 & \text { if there are } m \text { nodes at depth } z \text { in } \mathcal{G}_{t}, \\ 0 & \text { otherwise, }\end{cases}$

where $m \in\left\{1, \ldots, o_{\max }^{\mathcal{G}_{t}}\right\}$. We introduce $o_{\max }^{\mathcal{G}_{t}}$ hypotheses $\mathcal{H}_{m}, m=1, \ldots, o_{\text {max }}^{\mathcal{G}_{t}}: \mathcal{H}_{m}$ postulates that there are $m$ cell nuclei associated to the graph $\mathcal{G}_{t}$. A measure of conceivableness $\mathcal{M}_{m}$ is defined for each hypothesis as $\mathcal{M}_{m}=$ $\sum_{z} \mathbf{1}\left(o_{z}^{\mathcal{G}_{t}}=m\right)$. The hypothesis with the highest measure of conceivableness is finally chosen, and the associated number of nodes $m^{\star}$ gives the number of new graph(s) to build from $\mathcal{G}_{t}$. The new graph(s) is(are) directly obtained by a modification of $\mathcal{G}_{t}$, depth by depth. At depth $z$, if $o_{z}^{\mathcal{G}_{t}}>m^{\star}$, the $m^{\star}-o_{z}^{\mathcal{G}_{t}} \operatorname{node}(\mathrm{s})$ with the closest local maxima are merged. On the other hand, if $o_{z}^{\mathcal{G}_{t}}<m^{\star}$, the $o_{z}^{\mathcal{G}_{t}}-m^{\star}$ edge(s) with the highest cost within the edges that are linked to a same node is(are) removed. An example is illustrated in Fig.3 (c). 


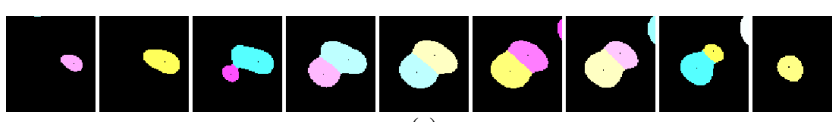

(a)

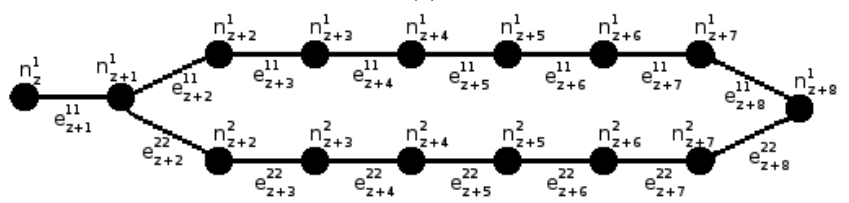

(b)

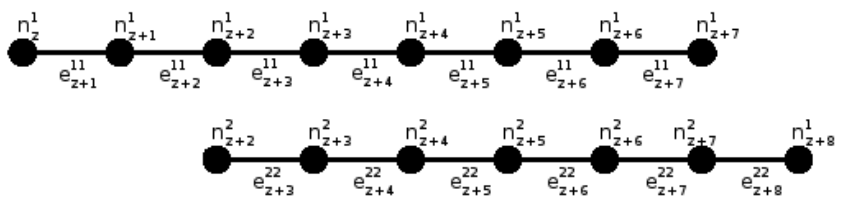

(c)

Fig. 3. (a) Nine consecutive cropped detection images where the different cell nucleus components are displayed in different colors; (b) graph associated with cell nucleus components in images (a); (c) final decision pertaining to the graph to characterize the two cell nuclei in images (a).

\section{INTENSITY PROJECTION BASED CORRECTION}

If two cell nuclei are proximal along depth, the decision process will fail. To correct this phenomenon, we enforce separation based on an intensity projection criterion.

As a consequence of their ellipsoidal shape, the projection along depth of a cell nucleus is an ellipse while the projection of two proximal cell nuclei results in a different shape (see Fig.4 (a;d)). Moreover, the average depth occupied by each pixel belonging to the cell nuclei shows a clearly different pattern when several cell nuclei are merged (see Fig.4 (b;e)). Let $\mathcal{C}=\left\{\mathbf{c}_{z}, \ldots, \mathbf{c}_{z+w}\right\}$ be a cell nucleus where $\mathbf{c}_{z}=\left\{c_{z}^{1}, \ldots, c_{z}^{q_{z}}\right\}$ is the set of $q_{z}$ points belonging to the cell nucleus at depth $z$. Let $\mathbf{p}=\left\{p^{1}, \ldots, p^{q}\right\}$ be the $q$ points corresponding to the projection of $\mathcal{C}$ along depth. By using the indicator function $\mathbf{1}(\cdot)$, we define the mean depth for each point in $\mathbf{p}$ :

$$
\left.M D\left(p^{i}\right)=\frac{\sum_{z} z \mathbf{1}\left(p^{i} \in \mathbf{c}_{z}\right)}{\sum_{z} \mathbf{1}\left(p^{i} \in \mathbf{c}_{z}\right.}\right) .
$$

The histogram computed from $M D$ represents the nucleus spatial distribution along depth and allows to identify when several cell nuclei are merged (see Fig.4). If this histogram shows more than one local maximum, several cell nuclei are merged. To separate them, we first consider that only two cell nuclei are merged and extract the first and last local maxima $\left\{z_{l m}^{1}, z_{l m}^{2}\right\}$ from the histogram. These two local maxima represent the depth boundaries for the new cell nuclei: above $z_{l m}^{1}$, all components correspond to the first cell nucleus while below $z_{l m}^{2}$, all components correspond to the second one. Three spatial regions can be extracted from $M D$ : a region only associated to the first cell nucleus (\#1 in Fig.4 (e)), a region only associated to the second cell nucleus (\#3 in Fig.4 (e)), and a region shared by the two cell nuclei (\#2 in fig.4 (e)). From the first two regions, we compute the centers-ofmass $\left\{p_{l m}^{1}, p_{l m}^{2}\right\}$. Between $z_{l m}^{1}$ and $z_{l m}^{2}$, all the points closer

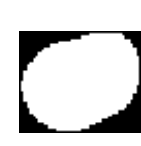

(a)

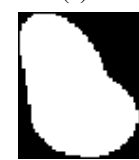

(d)

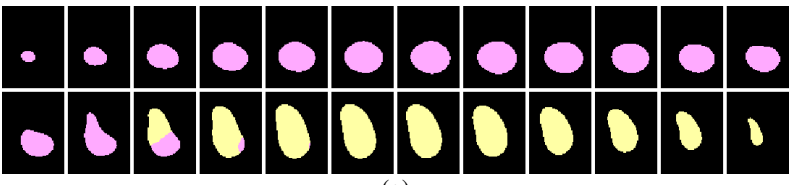

(g)

Fig. 4. (a) Projection of one cell nucleus along the depth direction; (b) mean depth computed from image (a); (c) histogram computed from image (b); (d) projection of the two cell nuclei along the depth direction; (e) mean depth computed from image (d); (f) histogram computed from image (e); $(\mathrm{g})$ separation of two cell nuclei shown in (d-e) with the corrective method applied duly.

\begin{tabular}{|c|c|c|c|c|c|c|c|c|c|c|}
\cline { 2 - 11 } \multicolumn{1}{c|}{} & \multicolumn{3}{c|}{ FPR } & \multicolumn{3}{c|}{ FNR } & \multicolumn{3}{c|}{ CSR } & N $_{0}$ \\
\hline & GDSM & LSSM & TSM & GDSM & LSSM & TSM & GDSM & LSSM & TSM & \\
\hline image \#1 & 0.08 & 0.01 & 0.01 & 0 & 0 & 0 & 0.87 & 0.91 & 0.92 & 153 \\
\hline image \#2 & 0.04 & 0.10 & 0.06 & 0.20 & 0.01 & 0.01 & 0.56 & 0.70 & 0.88 & 112 \\
\hline
\end{tabular}

Table 1. Results obtained with the GDSM, LSSM and TSM on images shown in Fig.5 (a) (image \#1) and in Fig.5 (e) (image \#2).

to $p_{l m}^{1}$ (resp. $p_{l m}^{2}$ ) belong to the first cell nucleus (resp. second cell nucleus). The same process is repeated until all the mean depth histograms only show one local maximum. An example is shown in Fig.4 (d-g).

\section{EVALUATION ON REAL DATA}

To evaluate the performances of our cell nuclei segmentation method, we consider two image volumes acquired at a resolution equal to $0.3 \times 0.3 \times 0.5 \mu \mathrm{m}^{3}$ (see Fig.5 (a)) and to $0.5 \times 0.5 \times 0.9 \mu \mathrm{m}^{3}$ (see Fig. 5 (e)). In order to face the two different resolutions, we use the $4^{\text {th }}$ wavelet plane for the first image and the $3^{\text {rd }}$ wavelet plane for the second image. We compare the results obtained with our Tracking based Segmentation method (TSM) against those obtained with a Gradient Diffusion based Segmentation method (GDSM) [5] and with a Level sets based Segmentation method (LSSM) [2], two non parametric methods that claim to be independent of the resolution. To quantitatively measure the accuracy of each method, an expert extracts from the results the number of false positives (NFP), false negatives (NFN) and correct segmentations (NCS). From these numbers, the ratios FPR $=\frac{\mathrm{NFP}}{\mathrm{N}_{0}}$, $\mathrm{FNR}=\frac{\mathrm{NFN}}{\mathrm{N}_{0}}$ and CSR $=\frac{\mathrm{NCS}}{\mathrm{N}_{0}}$ are defined, where $\mathrm{N}_{0}$ is the number of cell nuclei in the image volume. We do not consider the cell nuclei located at the image borders.

At a high resolution (image (a)), the GDSM tends to extract other structures than cell nuclei $(\mathrm{FPR}=0.08)$ while at a low resolution (image (e)), it misses $(\mathrm{FNR}=0.20)$ a large amount of cell nuclei, leading to a very low CSR (0.56). 


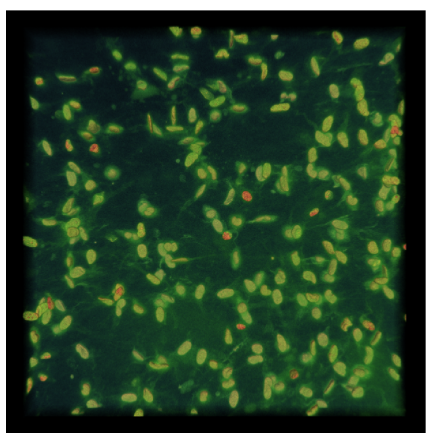

(a)

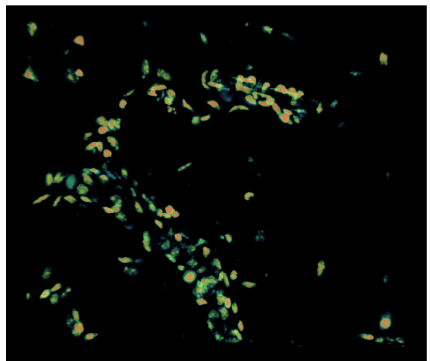

(e)

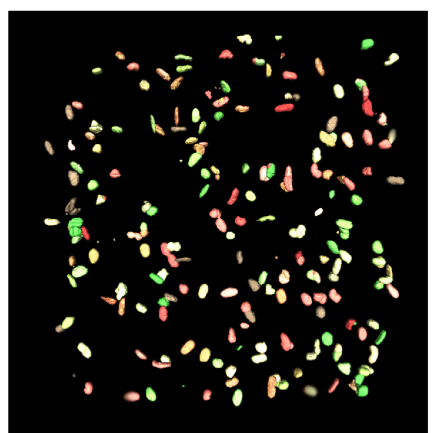

(b)

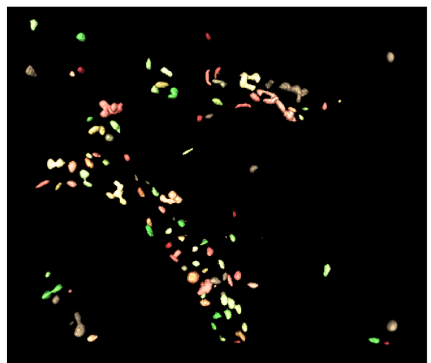

(f)

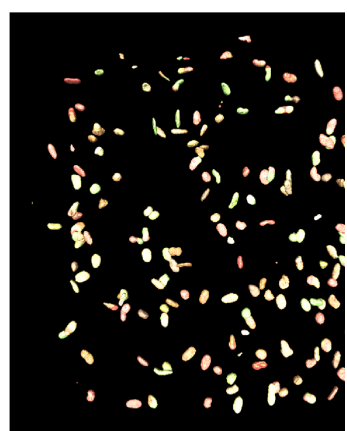

(c)

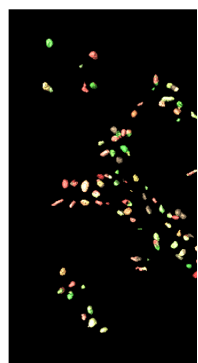

(d)

(h)

Fig. 5. (a) Volume rendering of a fluorescent confocal $1024 \times 1024 \times 105$ image acquired at a resolution equal to $0.3 \times 0.3 \times 0.5 \mu \mathrm{m}^{3}$; (b-d) surface renderings of cell nuclei segmentations obtained for image (a) using GDSM (b), LSSM (c) and TSM (d); (e) volume rendering of a fluorescent confocal $465 \times 400 \times 85$ image acquired at a resolution equal to $0.5 \times 0.5 \times 0.9 \mu \mathrm{m}^{3}$; (f-h) surface renderings of cell nuclei segmentations obtained for image (e) using GDSM (f), LSSM (g) and TSM (h).

These results show the dependence on a high resolution for the GDSM to obtain satisfying results. If the LSSM provides correct results at a high resolution, it shows a high FPR $(0.10)$ at a lower resolution. However, it still provides a better segmentation $(\mathrm{CSR}=0.7)$ than the GDSM. Although the FPR $(+0.05)$ obtained with the TSM increases when the resolution decreases, the results are similar at the two different resolutions. Overall, the obtained CSR (0.88) at a low resolution completely outperforms the CSR obtained with the GDSM $(+0.32)$ and the LSSM (+0.18).

\section{CONCLUSION}

In this paper, we propose a new 3D cell nuclei segmentation method based on detection of cell nuclei at each image plane followed by a tracking over depth. This method yields robust results for both isolated and cluttered cell nuclei over different image resolutions comparing to several existing methods. In practice, a key feature of this method is that it only requires the scale parameter that is derived from the image resolution, allowing robust use by non experts on a very large amount of images. This method is currently being applied to data from a breast tumor microenvironment study to elucidate the distribution of different cellular phenotypes in mouse models of different genotypes. For future work, we plan to test our method on a wider range of image volumes.

\section{Acknowledgement}

T. Pécot is recipient of the Pelotonia Fellowship Award.
7. REFERENCES

[1] A. Dufour, V. Shinin, S. Tajbakhsh, N. Guillen, J.-C. Olivo-Marin, and C. Zimmer, "Segmenting and tracking fluorescent cells in dynamic 3-D microscopy with coupled active surfaces," IEEE Trans. Image Process., vol. 14, fluorescent cells in dynamic
no. 9, pp. 1396-1410, 2005 .

[2] K. Mosaliganti, L. Cooper, R. Sharp, R. Machiraju, G. Leone, K. Huang, and J. Saltz, "Reconstruction of cellular biological structures from optical microscopy data," IEEE Trans. on Vis. and Comp. Graphics, vol. 14, no. 4, pp. 863-876, 2008.

[3] G. Lin, U. Adiga, K. Olson, J.F. Guzowski, C.A. Barnes, and B. Roysam, "A hybrid 3D watershed algorithm incorporating gradient cues and object models for automatic segmentation of nuclei in confocal image stacks," Cytometry A., vol. 56, no. 1, pp. 23-36, 2003.

[4] J. Cheng and J.C. Rajapakse, "Segmentation of clustered nuclei with shape markers and marking function," IEEE Trans. on Biomedical Engin., vol. 56, no. 3, pp. 741-748, 2009.

[5] G. Li, T. Liu, A. Tarokh, J. Nie, L. Guo, A. Mara, S. Holley, and S. Wong, "3D cell nuclei segmentation based on gradient flow tracking," BMC Cell Biology, vol. 40, no. 8, pp. http://www.biomedcentral.com/1471-2121/8/40, 2007

[6] D. Thomann, D.R. Rines, P.K. Sorger, and G. Danuser, "Automatic fluorescent tag detection in 3D with superresolution: application to the analysis of chromosome movement," Journal of Microscopy, vol. 208, Oct. 2002.

[7] D. Sage, H. Hediger, S.M. Gasser, and M. Unser, "Automatic tracking of particles in dynamic fluorescence microscopy," in Proceedings of the Third International Symposium on Image and Signal Processing and Analysis, Sept. 2003, pp. I.582-I.586.

[8] T. Pécot, A. Chessel, S. Bardin, J. Salamero, P. Bouthemy, and C. Kervrann, "Conditional random fields for object and background estimation in fluorescence video-microscopy," in Int. Symp. on Biomedical Imaging, Boston, 2009, pp. 734-737.

[9] S.R. Sternberg, "Biomedical image processing," IEEE Computer, vol. 16, no. 1, pp. 22-34, Jan. 1983

[10] L. Vincent, "Morphological grayscale reconstruction in image analysis: Applications and efficient algorithms," IEEE Transactions on Image Processing, vol. 2, no. 2, pp. 176-201, 1993.

[11] A. Chessel, B. Cinquin, S. Bardin, C. Kervrann, and Salamero J., "Computational geometry-based scale-space and modal image decomposition: Application to light video-microscopy imaging," in Conference on Scale Space and modal image decomposition: Application to light video-micros
Variational Methods, Voss, June 2009, vol. 5567, pp. 770-781.

[12] J.C. Olivo-Marin, "Extraction of spots in biological images using multi-scale products," Pattern Recognition, vol. 35, pp. 1989-1996, 2002

[13] J.L. Starck, J. Fadili, and F. Murtagh, "The undecimated wavelet decomposition and its reconstruction," Image Processing, vol. 16, no. 2, pp. 297-309, 2007.

[14] P.K. Sahoo, D.W. Slaaf, and T.A. Albert, "Threshold selection using a minimal histogram entropy difference," Society of Photo-Optical Instrumentation Engineers, vol. 36, Mar. 1997.

[15] L.D. Cohen and R. Kimmel, "Global minimum for active contour models: A minimal path approach," Internation Journal of Computer Vision, vol. 24, pp. 57-78, Aug. 1997.

[16] V. Racine, M. Saschse, J. Salamero, V. Fraisier, A. Trubuil, and J.B. Sibarita, "Visualization and quantification of vesicle trafficking on a 3D cytoskeleton network in living cells," Journal of Microscopy, pp. 214-228, Mar. 2007.

[17] L.D. Cohen, "Minimal paths and fast marching methods for image analysis," Mathematical Models in Computer Vision: the Handbook, 2005. 